\title{
Commentary
}

\section{Building for the Known Unknown-Development of the National Centre for Infectious Diseases}

Eugene Fidelis $\underline{\text { Soh, }}{ }^{1} M B B S$ MPH

In the midst of the COVID-19 pandemic, an interesting question arose as to why the wards at the National Centre for Infectious Diseases (NCID) were similar in design to those at the main Tan Tock Seng Hospital (Main Hospital) (Fig. 1). This same question had been asked at the onset of the planning and design for NCID more than 10 years ago. The deliberate similarities in the design of the wards at NCID to those in the Main Hospital enabled integrated operations, continuing innovation and surge capabilities. In building NCID, Tan Tock Seng Hospital (TTSH) was mindful that she would have to transition her workforce and resources from business-as-usual (BAU) tertiary care to an outbreak response in a matter of hours and days.

\section{Preparedness for the Unknown}

Following the Severe Acute Respiratory Syndrome (SARS) outbreak in 2003, ${ }^{1}$ an early plan was in the works to replace TTSH's Communicable Disease Centre $(\mathrm{CDC})^{2}$ with a new facility, then nicknamed "ABC". In 2009, TTSH was called upon to respond to another outbreak of H1N1 Influenza. For the second time in her recent history, the hospital rushed to build a tent outside of her Emergency Department, set up makeshift gantries to restrict visitors to her wards, and shut down the hospital to respond to the outbreak. While the H1N1 outbreak was relatively mild compared to SARS, it was a wake-up call that lessons learnt had to be incorporated into the hospital's facilities.

TTSH was tasked to redevelop the CDC as a national asset with a national public health role overseen by the Ministry of Health (MOH), Singapore. The new facility was to be integrated with TTSH for clinical operations and support. It was to function as a tertiary hospital for infectious diseases (ID), working closely to develop and support ID services, education and research across Singapore. The redevelopment of CDC as NCID is often attributed to lessons learnt from SARS. ${ }^{3}$ In the initial planning stages for NCID, the hospital sought to incorporate lessons to contain an outbreak similar to that of SARS. ${ }^{2}$ But the H1N1 outbreak taught her that no 2 outbreaks would be the same. With novel pathogens and what was known about outbreaks, the hospital had to build NCID for the 'known unknown'. Soon after H1N1, assumptions were revisited and it was clear that the hospital needed flexibility in the capacity and design of the new outbreak facility.
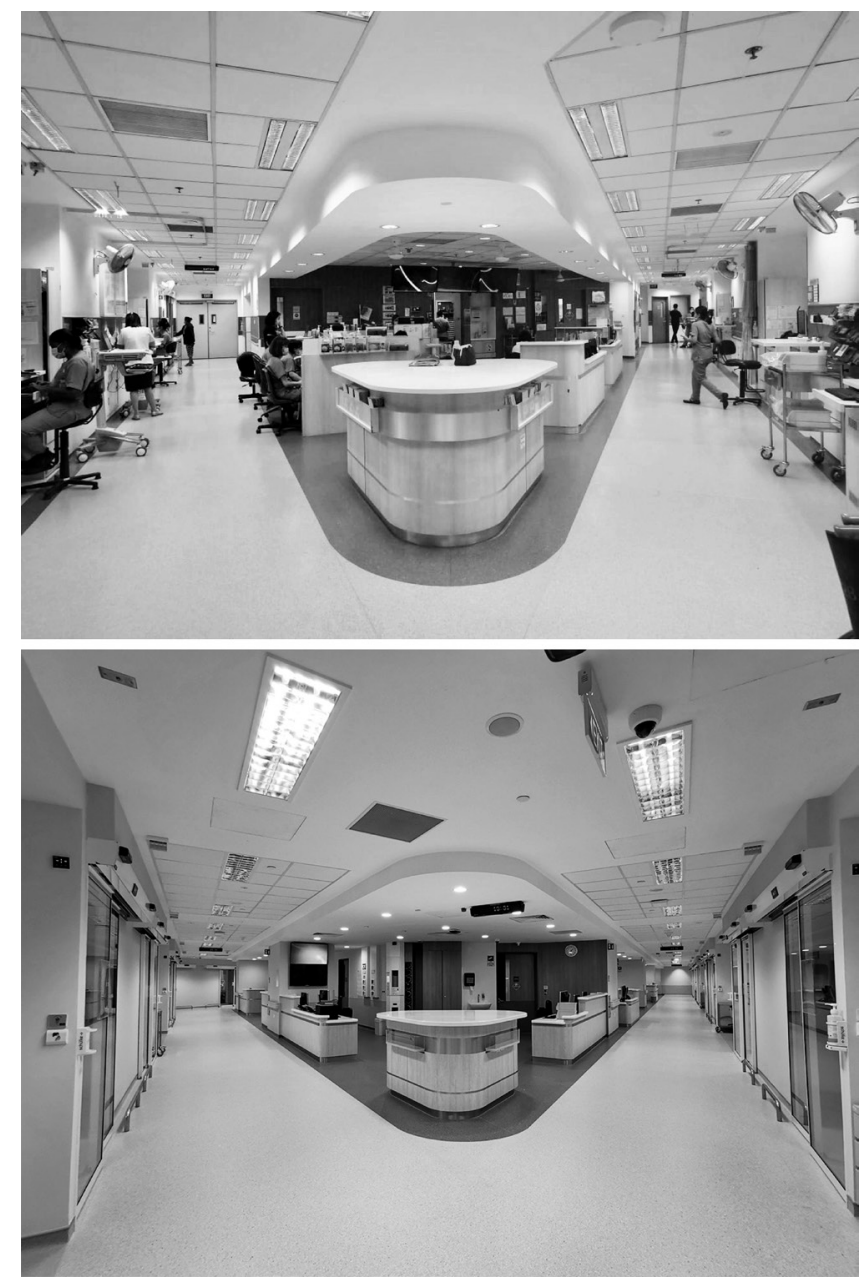

Figure 1. TTSH Ward (top) and NCID Ward (bottom) follow a similar design.

${ }^{1}$ Tan Tock Seng Hospital, National Healthcare Group, Singapore

Address for Correspondence: Dr Eugene Fidelis Soh, Tan Tock Seng Hospital, 11 Jalan Tan Tock Seng, Singapore 308433

Email: eugene_fidelis_soh@ttsh.com.sg 


\section{Planning for the Future}

With MOH's support in 2010, TTSH drew up an integrated healthcare master plan to build Singapore's third medical hub-HealthCity Novena. The master plan was a blueprint of how TTSH would anchor the health of our population living in Central Singapore through key developments up to 2030. The development of healthcare facilities is inherently "lumpy". Long-term healthcare planning is essential to meet the needs of a fast-ageing population, and to integrate care across healthcare facilities.

The 17-hectare land use was approved by the Urban Redevelopment Authority, and launched by Minister for Health, Mr Gan Kim Yong, on 30 August 2013 as HealthCity Novena Master Plan 2030.4,5 A plot of land directly opposite the Main Hospital was earmarked for NCID. It was an awkward teardrop-shaped land parcel with a narrow width and sloping gradient. Although the physical constraints of the site were challenging, the location (next to the Main Hospital) was perfect.

There were 3 key considerations in selecting a site next to the Main Hospital. Firstly, NCID will not operate as a standalone hospital. Secondly, NCID needs to be ramped up quickly during an outbreak. Thirdly, NCID requires augmentation of multi-disciplinary specialist care, inter-professional team-based care, a large 24/7 clinical laboratory, and the assurance of a strong supply chain and support operations. Patient care at NCID is more akin to a tertiary hospital than a single specialty centre.

Various alternative sites were suggested for NCID. There had been a suggestion that NCID was best located far from population centres. This was a mental model of the past, when treatment was basic and facilities were built mainly for quarantine purposes. With the advancement of medicine and engineering, NCID stands as a treatment and isolation facility, built to the highest safety standards to enable the facility to be located in an urban area.

Also incorporated into NCID are the National Public Health Laboratory (NPHL), National Public Health and Epidemiological Unit(NPHEU), Antimicrobial Resistance Coordinating Office and various national public health programmes. This allows for an integrated public health and clinical response to be mounted in any outbreak response led by $\mathrm{MOH}$.

\section{Building to Flow and Function}

The Main Hospital at TTSH was rebuilt from ground-up and completed in 1999. The building featured a revolutionary triangular ward design with 4 wards on every floor; organised in a butterfly-shaped configuration with a ward on each wing and a central core for support. The triangular layout maximises line of sight of patients. Two arms of the triangular ward cater to patient care whereas the third arm provides for support services.

To begin the infrastructural planning for NCID, a base floor layout was needed for a stackable design that could be fitted into the narrow plot of land. TTSH adopted the Main Hospital's triangular ward design as the base floor layout for NCID. The primary reason to adopt the same shape was the need for TTSH staff to be familiar with the ward layout when deployed to NCID. Given the shape of the land, and the plan for 330 beds, the chosen design had 2 triangular wards on each floor in a rhombus shape that later became more of a parallelogram.

A Production Preparation Process (3P) Lean design project ${ }^{6}$ was conducted to ensure flows were segregated for patients, staff, visitors, supplies and such. A 3-tier "design to flow and function" approach was adopted. The first tier was a "Macro-3P" design which dealt with the overall hospital flow. The next tier was a "Micro-3P" design to ensure flow and functions within the ward, while the last tier was the "Nano-3P" design that dealt with the furniture and fittings to optimise the tasks to be done within a room or specialised space. After many design workshops and mock-ups, one can say that NCID was built ground-up by users including doctors, nurses, allied health and operations.

\section{Designing on Principles to be Flexible}

When dealing with a novel pathogen, there can be no fixed assumptions. It was clear that with learnings from the previous outbreaks, ${ }^{7}$ TTSH had to put together what was known, to build NCID for the unknown. Therefore, in building for known unknown, a set of design principles $^{5}$ was adopted for the new outbreak facility to ensure connectivity, safety, scalability, capability, and convertibility. 


\section{NCID and TTSH Connectivity}
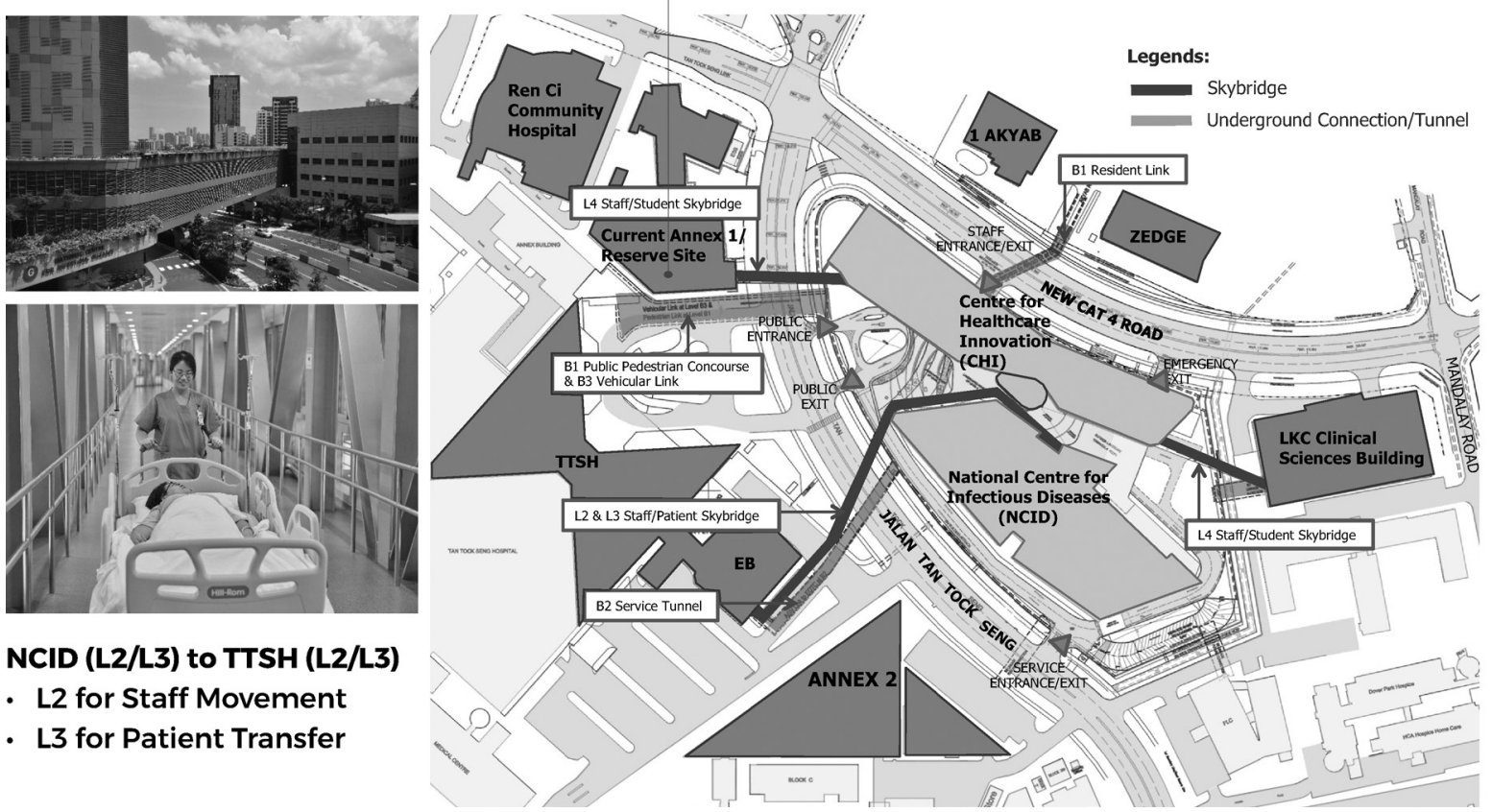

Figure 2. Connectivity Between TTSH and NCID.

\section{Connectivity}

NCID was designed to segregate flows within its building, and with the Main Hospital (Fig. 2). It is connected via a double-decked bridge to the TTSH Emergency Block, providing direct access for transfers of patients. This allows for clean and dirty flows to be segregated on 2 separate decks. The bridge also has direct access to lift lobbies within NCID for inter-floor transfers. Underground, it is connected via 3 segregated tunnels for visitors, cars and supplies. The supplies tunnel is run by automated guided vehicles which transport supplies to and from the Main Hospital. Two other bridges connect to the adjacent building, the $\mathrm{Ng}$ Teng Fong Centre for Healthcare Innovation (CHI), which shares a common podium with NCID. This allows for continuing campus connectivity by bypassing NCID during an outbreak.

\section{Safety}

Besides segregating flows within NCID for the safety of staff, visitors and patients, it is important that NCID is safe for her neighbours in a densely populated area. The $\mathrm{CHI}$ building provides a visual buffer between NCID and the nearby residential estate. The Mechanical and Electrical system for NCID is designed to exceed existing international recommendations for hospitals managing infectious diseases. For NCID wards, the airflow design is a purpose-built, single-pass ventilation system without re-circulation, with separate Air Handling Units. Its exhaust air passes through the top-tier High Efficiency Particulate Air filters $(99.999 \%)$, and is treated with ultraviolet rays to eliminate microorganisms, before dispersing into the atmosphere with a high plume fan at the rooftop. The 
wards feature Negative Air Pressure Rooms, tested for air-tightness, which ensures safety of staff and patients. A specially designed High-Level Isolation Unit (HLIU) caters to highly-virulent infectious diseases such as haemorrhagic fevers (e.g. Ebola), possible bio-threats (e.g. smallpox) and novel pathogens. The HLIU is a bio-containment unit that has a dedicated laboratory and self-decontamination facilities.

\section{Scalability}

NCID needed to be scalable in design to meet different and fast-changing outbreak requirements. At the same time, over-sizing NCID would have increased operating costs during non-outbreak periods. While we planned for 330 beds based on SARS, no 2 outbreaks are the same, and the population size will grow. So, the design of NCID was future-proofed to be able to increase the number of beds to 586, with ready oxygen points. Scalability of beds requires augmentation of ready manpower. The wards at NCID and the Main hospital had to be standardised in layout and processes to enable staff deployed from the Main Hospital to open outbreak wards at NCID at short notice ( 2 to 8 hours) and function immediately.

\section{Capability}

The opportunity to build NCID also allowed the introduction of new capabilities. At the systems level, a new TTSH Operations Command Centre (OCC) was sited at $\mathrm{CHI}$ to remain accessible if NCID or the Main Hospital were to be locked down during an outbreak. The OCC was developed to integrate real-time operations between our Main Hospital and NCID. Integrated operations enable wards at the Main Hospital to be decanted to transfer manpower to NCID in order to open new outbreak wards. Regular patients warded at NCID would have to be transferred back to the Main Hospital as well. A new Command, Control and Communications (C3) system akin to the brain of the hospital, was introduced at OCC to provide real-time hospital flow and resource management. This enabled careful sequencing, and optimisation of hospital operations, to ensure timely outbreak response.

Another new capability introduced at NCID was a real-time location system (RTLS) using WiFi. RTLS was piloted at NCID for contact tracing and hand hygiene monitoring. While RTLS has been used for tracking general locations, we needed to achieve a 2-metre tag-to-tag sensing capability for contact tracing. The RTLS also enables staff and patient movements to be studied to optimise workflows. Other capabilities include the NPHL, which is a Bio-Safety Level 3 laboratory situated on the top floor of NCID.

\section{Convertibility}

A space was designed at NCID that could be converted to a screening centre (SC) for outbreaks. The SC can be separated into 5 zones for scalability and risk management, with on-site radiology services, pharmacy store, and nearby satellite laboratory testing. The SC has isolation rooms and open spaces that are flexible in configuration. Another key aspect of convertibility is that all inpatient rooms are standardised to allow for different room use: isolation, cohorting or intensive care (Fig. 3). Demountable walls can be installed in days to convert cohort rooms into negative pressure isolation rooms if necessary. This gives NCID the flexibility of both bed types and numbers to respond more effectively to the wide ranging requirements of different pathogens and different phases of an outbreak.

\section{Lessons Learnt and Relearnt}

The COVID-19 pandemic has tested the effectiveness of these design principles to enable TTSH and NCID to respond at pace and scale according to the outbreak situation. During non-outbreak operations, 150 beds at NCID were used for BAU patients with infectious diseases and overflow from the Main Hospital. On 28 January 2020, with escalating numbers of COVID-19 patients, TTSH was activated to scale NCID progressively to 330 beds. By 7 February 2020, when DORSCON $^{8}$ (Disease Outbreak Response System Condition) Orange was declared by MOH, NCID was already in full operations at 330 beds. With MOH's approval on 15 March 2020, NCID was further augmented by TTSH and other Public Healthcare Institutions (PHI) to increase its built-in contingency to 586 beds. To operate at 586 beds, a total of 1,688 headcounts (including 107 headcounts from other PHIs) were deployed to NCID to augment its manpower base of 687. At the peak of the outbreak response, TTSH operated up to 1,475 beds for COVID-19, including the 586 beds at NCID. Overall, the flexible design for NCID, its co-location and integrated operations with the Main Hospital, and the ability to quickly transfer manpower enabled TTSH and NCID to respond effectively.

There are new lessons learnt, arising from the COVID-19 outbreak for NCID with respect to its facilities design. Firstly, NCID's capacity was designed for a medium-sized outbreak, and even with its added contingency, its capacity was insufficient for a larger outbreak like COVID-19. Its location next to, and 


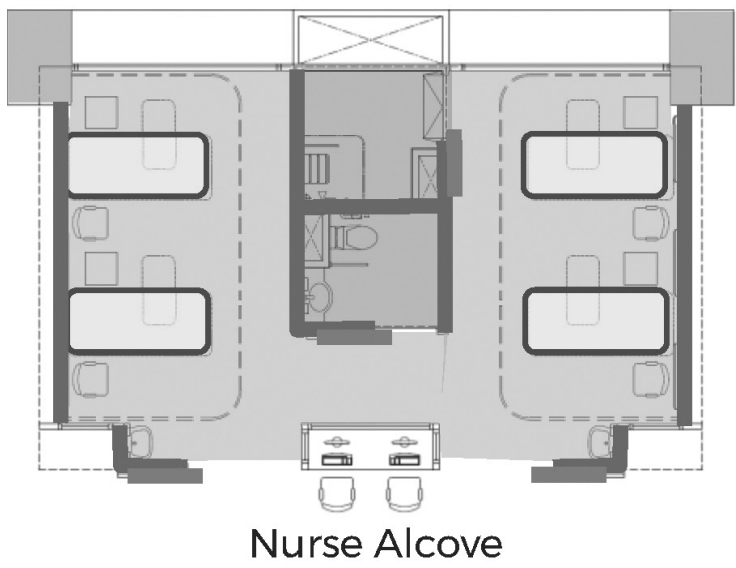

COHORT

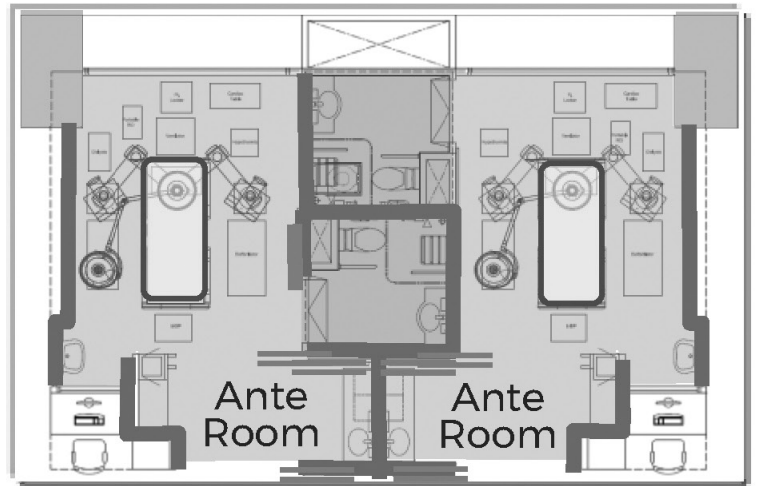

Nurse Alcove
Nurse Alcove

INTENSIVE CARE UNIT

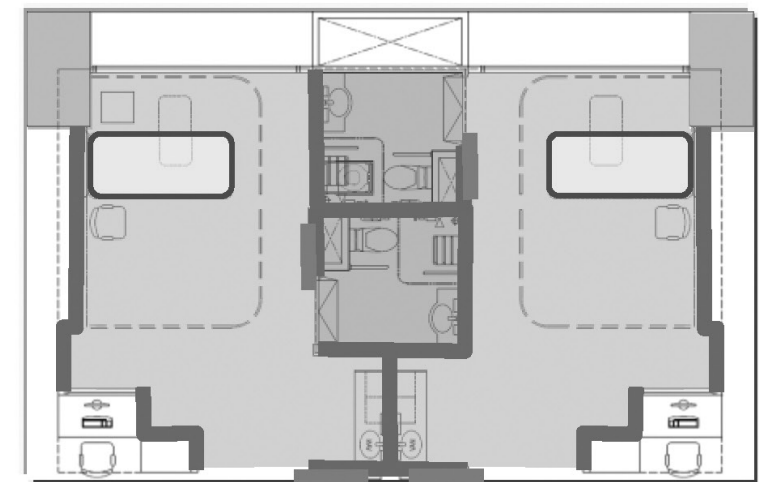

Nurse Alcove
ISOLATION

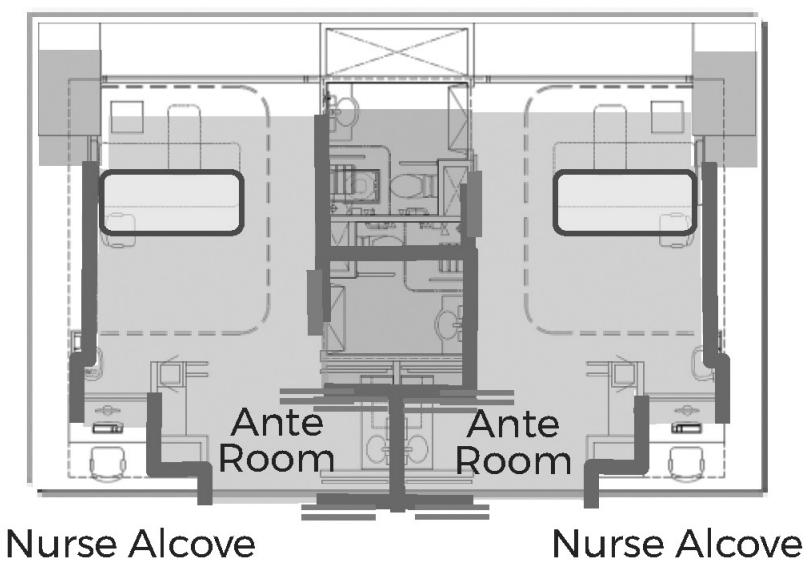

NEGATIVE PRESSURE

Figure 3. Schematic illustration of the Convertibility of Patient rooms in the NCID Screening Centre.

connection with, the Main Hospital enabled a quick campus ramp-up of bed capacity beyond NCID. A National Public Health response led by $\mathrm{MOH}$ enabled other PHIs to ramp up bed capacity to further meet outbreak demand, with NCID providing ID leadership across the system. The opening of government quarantine facilities, further augmentation by private hospitals and set up of large-scale community care facilities for recovering patients, ${ }^{9}$ enabled $\mathrm{MOH}$ to conserve bed capacity at PHIs for those who required hospital care. Secondly, NCID's screening centre had to be expanded with additional tents as the safe distance between patients was increased from 1 metre to 2 metres based on infection control requirements. The outpatient clinic above the screening centre was planned as a contingency overflow site, but it did not feature single pass air exchange. Hence, the use of tents to expand the screening centre during this outbreak. Thirdly, the installation of demountable walls required specialist manpower at short notice and was subject to 
availability during an outbreak. The installation, which took approximately 5 days per ward, also meant critical downtime during an outbreak. These lessons and others, going forward, allow for further improvements to NCID's facilities design and contingency plans.

\section{Conclusion}

NCID was officially opened by Minister for Health, Mr Gan Kim Yong, on 7 September 2019. Shortly after, Singapore reported her first case of imported COVID-19 on 23 January 2020. NCID had opened just in time. Today, we are better prepared and have a purpose-built facility that allows us to better manage surges in an outbreak. While there are limits to what NCID can achieve as a facility, she has been built flexible for the known unknown. NCID stands at the vanguard of Singapore's outbreak response, with the Main Hospital as its hinterland of support and never a bridge too far away. NCID will always be a work-inprogress as the hospital learns from every outbreak. This has been the good work of generations and will be the continuing work for generations to come.

\section{REFERENCES}

1. Ng WC. The Silent War. Singapore: Tan Tock Seng Hospital; 2004.

2. Mohammad Raji KS, Hsu LY, Loh KS. The Communicable Disease Centre and Challenges in Infectious Disease Management in Singapore. Ann Acad Med Singapore. 2020 Feb;49(2):88-92. PMID: 32246710 .

3. Tai DY. SARS: how to manage future outbreaks? Ann Acad Med Singapore. 2006 May;35(5):368-73. PMID: 16830006.

4. Khalik S. Massive Health-Care Complex by 2030. The Straits Times. 2013 Aug 31.

5. Write Editions. HealthCity Novena-Building a Community of Care. Singapore: HealthCity Novena, Development Office, Tan Tock Seng Hospital; 2019.

6. Chan CC, Wiggins B. The Development of a Lean Concept (3P) for the Proposed National Centre for Infectious Diseases (NCID), Singapore. Singapore: Simpler; 2011 Oct. p 6-9.

7. Chen M, Leo YS, Ang B, Heng BH, Choo P. The outbreak of SARS at Tan Tock Seng Hospital-relating epidemiology to control. Ann Acad Med Singapore. 2006 May;35(5):317-25. PMID: 16829998.

8. Ministry of Health, Singapore. MOH Pandemic Readiness and Response Plan for Influenza and other Acute Respiratory Diseases. Singapore: MOH; Revised April 2014.

9. Ministry of Health, Singapore. Comprehensive Medical Strategy for COVID-19. Singapore: MOH Press Release. 2020 Apr 28. 